\title{
Periodontitis and Prevalence of Elevated Aminotransferases in the Hispanic Community Health Study/Study of Latinos
}

\author{
Aderonke A. Akinkugbe ${ }^{\star}$, A. Sidney Barritt ${ }^{\dagger}$, Jianwen Cai ${ }^{\ddagger}$, Steven Offenbacher ${ }^{\S}$, Bharat \\ Thyagarajan $\|$, Tasneem Khambaty $\rrbracket$, Richard Singer\#, Eric Kallwitz ${ }^{* *}$, Gerardo Heiss ${ }^{\dagger \dagger}$, and \\ Gary D. Slade \\ *Oral Health Services Research Core, School of Dentistry, Virginia Commonwealth University, \\ Richmond, VA \\ †Department of Gastroenterology and Hepatology, University of North Carolina at Chapel Hill, \\ Chapel Hill, NC \\ ‡Department of Biostatistics, University of North Carolina at Chapel Hill, Chapel Hill NC \\ $\S$ Department of Periodontology, University of North Carolina at Chapel Hill, Chapel Hill, NC \\ |Department of Laboratory Medicine and Pathology, University of Minnesota, Minneapolis, MN \\ IDepartment of Psychology, University of Maryland, Baltimore County, Baltimore MD \\ \#Department of Public Health Sciences, University of Miami Miller School of Medicine, Miami, FL \\ and Nova Southeastern University, College of Dental Medicine, Ft. Lauderdale, FL \\ **Department of Medicine, Loyola University Medical Center, Maywood IL \\ ${ }^{+}$Department of Epidemiology, University of North Carolina at Chapel Hill, Chapel Hill, NC \\ ¥¥Department of Dental Ecology, University of North Carolina at Chapel Hill, Chapel Hill, NC
}

\section{Abstract}

Objective-NAFLD prevalence is greater among Hispanics/Latinos than other racial/ethnic groups and is reported to vary among Hispanic/Latino groups. Experimental animal and human studies demonstrate associations between periodontitis and non-alcoholic fatty liver disease (NAFLD), not yet reported among Hispanics/Latinos. This study examined periodontitis as a novel risk factor that may contribute to the burden of NAFLD among Hispanics/Latinos.

Methods-Data came from 11,914 participants of the Hispanic Community Health Study/Study of Latinos. Periodontitis was defined as the extent (none, <30\%, $230 \%$ ) of periodontal sites with clinical attachment level (CAL) of $\geq 3 \mathrm{~mm}$ or probing pocket depth (PD) of $\geq 4 \mathrm{~mm}$. Elevated serum

Author for Correspondence: Aderonke A. Akinkugbe, Oral Health Services Research Core, School of Dentistry, Virginia Commonwealth University.1101 E. Leigh Street, Richmond VA, 23298-0566. Telephone: (804) 628-7616. aaakinkugbe@ vcu.edu. Authors declare no conflicts of interest with respect to the authorship and/or publication of this article.

Disclosures: Authors have no conflicts to disclose

Author contributions: AAA, ASB, SO, GH, GDS- study concept and design; acquisition of data; analysis and interpretation of data; drafting of the manuscript; critical revision of the manuscript for important intellectual content; statistical analysis; obtained funding; administrative, technical, or material support; study supervision JC, BT, RS, EK- critical revision of the manuscript for important intellectual content; statistical analysis and interpretation of results. 
transaminases indicative of suspected NAFLD were defined as having alanine aminotransferase levels $($ ALT) $>40$ IU/L or aspartate aminotransferase $($ AST) $>37$ IU/L for men and ALT >31 IU/L or AST > 31 IU/L for women. Survey-logistic regression models estimated prevalence odds ratios (POR) and $95 \%$ confidence intervals (CI) of the association between periodontitis and suspected NAFLD.

Results - The overall age standardized percentage of study participants with $<30 \%$ of sites with CAL $\geq 3 \mathrm{~mm}$ or PD $\geq 4 \mathrm{~mm}$ was $53.5 \%$ and $58.6 \%$ respectively, while those with $230 \%$ sites with $\mathrm{CAL} \geq 3 \mathrm{~mm}$ or PD $\geq 4 \mathrm{~mm}$ comprise $16 \%$ and $5.72 \%$ respectively. The overall age-standardized prevalence $(95 \% \mathrm{CI})$ of suspected NAFLD was $18.1 \%(17.1 \%-19.0 \%)$. Within the entire cohort, we observed a dose-response association between PD $\geq 4 \mathrm{~mm}$ and the prevalence odds of suspected NAFLD, whereby participants with $<30 \%$ affected had a crude POR $=1.19$ (95\% CI: $1.03,1.38$ ) while those with $230 \%$ affected had a crude POR $=1.39$ (95\% CI: $1.02,1.90)$. These crude estimates were attenuated towards the null and rendered non-significant upon covariate adjustment. No differences were found by Hispanic/Latino background group.

Conclusion-Previously reported associations between periodontitis and NAFLD were marginal to null in this study of a diverse group of Hispanics/Latinos.

\section{Keywords}

HCHS/SOL; Epidemiologic studies; Periodontitis; Transaminases; Cross-sectional Studies

\section{Introduction}

Non-alcoholic fatty liver disease (NAFLD) is the most common type of liver disease and the hepatic component of the metabolic syndrome. ${ }^{1,2}$ Estimated to affect $17-33 \%$ of U.S. adults, ${ }^{1-4}$ NAFLD is associated with higher healthcare costs and mortality. ${ }^{1,5,6}$ Insulin resistance and obesity, both characterized by chronic low-grade systemic inflammation $1,7,8$ are identified as NAFLD risk factors. Indeed, epidemiologic studies have reported associations between pro-inflammatory cytokines and components of the metabolic syndrome ${ }^{9}$ including NAFLD. ${ }^{10,11}$ Thus, chronic health conditions that elicit systemic inflammatory response potentially represent unrecognized but potentially modifiable factors in the etiology of NAFLD. Specific to the Hispanic/Latino population, NAFLD prevalence is reported to be greater compared to estimates reported for non-Hispanic Whites and AfricanAmericans. ${ }^{12,13}$ Furthermore, there is heterogeneity in reported NAFLD prevalence among Hispanics/Latinos, and risk factors such as genetics, lifestyle and environment as well acculturation do not completely account for the burden of disease in this population. ${ }^{12,13}$ Therefore, it is important to identify novel modifiable risk factors for NAFLD.

Periodontitis, a major cause of tooth loss 14,15 is a chronic inflammatory disease of the oral cavity, involving the gingiva, connective tissue and alveolar bone supporting the teeth. Affecting about $45 \%$ of the adult U.S. population, ${ }^{16,17}$ periodontitis has been shown in rat models to be causally related to ${ }^{18,19}$ and in epidemiologic studies to be associated ${ }^{20-24}$ with insulin resistance. Periodontitis has also been linked to low-grade systemic inflammation in experimental animal ${ }^{25,26}$ and epidemiologic ${ }^{20,27-30}$ studies. Recently, periodontitis was linked to NAFLD in both mice models ${ }^{31,32}$ and human studies, ${ }^{31,33}$ but the relationship has 
not been investigated in an ethnically diverse population group. Because NAFLD disproportionately affects Hispanics/Latinos and previous studies on the association between periodontitis and NAFLD have mostly focused on Caucasians ${ }^{33}$ and Asians, ${ }^{31}$ the objective of this study was to examine associations between periodontitis and indicators of NAFLD in a diverse group of Hispanic/Latino men and women who participated in the Hispanic Community Health Study/Study of Latinos (HCHS/SOL).

\section{Materials and Methods}

\section{Study population}

The HCHS/SOL is a multicenter community based cohort study of 16,415 self-identified Hispanic/Latino men and women, designed to investigate risk and protective factors for chronic health conditions. Eligible 18-74-year-olds of Cuban, Dominican, Mexican, Puerto Rican, Central and South-American background were recruited between March 2008 and June 2011 from randomly selected households in 4 U.S. communities (Bronx, New York; Chicago, Illinois; Miami, Florida; and San Diego, California) using a stratified two-stage area probability sampling. ${ }^{34,35}$ Oversampling of 45-74-year olds was conducted, and sampling weights were calculated to reflect this disproportionate sampling. At baseline, study participants completed interviewer-administered questionnaires, underwent clinical assessments including dental examination, and a sample of whole blood was collected for laboratory analysis. ${ }^{34-36}$ Institutional Review Boards of all participating institutions approved the study and all participants gave informed consent.

This study was restricted to participants who completed dental examinations with serum transaminase measurements, who did not have detectable serum levels of hepatitis B surface antigen or hepatitis $\mathrm{C}$ antibody. Furthermore, restricted to participants who did not report excessive alcohol consumption (consuming $>70 \mathrm{~g}$ of ethanol/week for women and $>140 \mathrm{~g} /$ week for men), and had transferrin saturation levels of $50 \%$.

\section{Exposure of interest}

Study participants not requiring antibiotic prophylaxis received a full-mouth periodontal examination following a standardized protocol. Measurements of probing pocket depth (PD) and gingival recession were recorded on 6 sites (mesio-buccal, mid-buccal, disto-buccal, mesio-lingual, disto-lingual, and lingual) per tooth except third molars. PD is the distance from the free gingival margin to the bottom of the sulcus while gingival recession is the distance from the cementoenamel junction to the free gingival margin. Clinical attachment level (CAL) was calculated as sum of PD and gingival recession. Examiners were recalibrated annually to a gold standard examiner, with very good to excellent agreement. ${ }^{37}$ This investigation defined periodontitis as the proportion of periodontal sites (none, $<30 \%$, $230 \%$ ) affected by CAL $\geq 3 \mathrm{~mm}$ or PD $\geq 4 \mathrm{~mm} .{ }^{38}$ Because this definition takes into consideration measurements made at all periodontal sites, it permits comparison to previous prevalence studies and is preferred to alternative classification systems that only uses the "worst score". 38 


\section{Outcome of interest}

Elevated serum transaminase levels indicative of suspected NAFLD were defined as having alanine aminotransferase (ALT) $>40 \mathrm{IU} / \mathrm{L}$ or aspartate aminotransferase (AST) $>37 \mathrm{IU} / \mathrm{L}$ for men and ALT $>31 \mathrm{IU} / \mathrm{L}$ or AST $>31 \mathrm{IU} / \mathrm{L}$ for women. ${ }^{39}$

The presence of NAFLD was also assessed with the fatty liver index (FLI), ${ }^{40}$ an algorithm based on BMI, waist circumference, triglycerides and gamma glutamyl-transferase levels (GGT) to indicate the likelihood of having NAFLD. This index based on the equation below is reported to be $84 \%$ accurate and a FLI score of $₫ 60 \%$ indicates a high likelihood of NAFLD. ${ }^{40}$

$$
\begin{aligned}
& \mathrm{FLI}=100 * \\
& \left(\frac{e^{(0.953 * \operatorname{loge}(\text { triglycerides })+0.139 * \mathrm{BMI}+0.718 * \operatorname{loge}(\mathrm{ggt})+0.053 * \text { waist circumference }-15.745)}}{1+e^{(0.953 * \log \mathrm{e}(\text { triglycerides })+0.139 * \mathrm{BMI}+0.718 * \operatorname{loge}(\mathrm{ggt})+0.053 * \text { waist circumference }-15.745)}}\right)
\end{aligned}
$$

Study participants with a FLI score $\measuredangle 60 \% 40$ were classified as having a high likelihood of NAFLD.

\section{Covariates}

Age and gender were self-reported. Waist circumference measured in centimeters $(\mathrm{cm})$ was dichotomized as $>88 \mathrm{~cm}$ for women and $>102 \mathrm{~cm}$ for men indicating the presence of abdominal obesity as part of the National Cholesterol Education Program, Adult Treatment Panel (NCEP ATP) III definition of the metabolic syndrome. Smoking was categorized into non-smoker, former and current smokers based on current and previous use of at least 100 cigarettes in a lifetime. Diabetes status was based on the American Diabetes Association definition, ${ }^{41}$ namely participants with normal and impaired fasting glucose or impaired glucose tolerance (fasting glucose between $100-125 \mathrm{mg} / \mathrm{dl}$ or post oral glucose tolerance test (OGTT) between 140-199 mg/dl or HbA1C between 5.7 and <6.5\%) were categorized as not having diabetes. Acculturation was evaluated using the six-item language subscale of the Short Acculturation Scale for Hispanics that characterizes language on a 5-point Likert scale. ${ }^{42}$ The scale is interpreted as 1 -Spanish only speakers, 2-speaks Spanish better than English, 3- speaks Spanish and English equally, 4- speaks English better than Spanish, and 5- English only speaker. ${ }^{42,43}$ Physical activity (PA) was calculated as the average of the total amount of time per week engaged in work or recreational physical activity and the total metabolic energy expenditure based on the Global Physical Activity Questionnaire. ${ }^{44}$ This variable was categorized into high [(vigorous work and recreational PA $\geq 3$ days and total metabolic expenditure (MET) $\geq 1,500$ ) or (moderate and vigorous PA $\geq 7$ days and MET $23,000)$ ], moderate [(vigorous work and recreational PA $\geq 3$ days and total vigorous activity level of $\geq 60$ minutes for 7 days or moderate work, transportation and recreational PA for $\geq 5$ days and total moderate activity level of $\geq 150$ minutes for 7 days or moderate and vigorous PA level for $\geq 5$ days and total activity level of $\geq 600$ minutes for 7 days)] and low physical activity level for those not meeting the criteria for high and moderate PA. Educational attainment was categorized as: <high school, high school, or > high school. 


\section{Statistical analysis}

Of the 16,415 HCHS/SOL participants, 11,914 were eligible for this study. Reasons for exclusions include: excessive alcohol consumption ( $n=828)$, missing ALT/AST ( $n=169)$, hepatitis B surface antigen and hepatitis $C$ antibody in blood samples $(n=356)$, transferrin saturation $>50 \%(n=690)$, no dental examination and/or edentulous $(n=1,275)$ and report of 'other' as Hispanic/Latino background $(\mathrm{n}=503)$. Also excluded were participants with missing data on the following covariates: diabetes $(n=21)$, alcohol use $(n=65)$, education $(\mathrm{n}=91)$, acculturation score $(\mathrm{n}=102)$, physical activity $(\mathrm{n}=140)$, smoking $(\mathrm{n}=93)$, and waist circumference $(n=74)$.

Weighted proportions and standard errors (SE) for the study population characteristics were estimated for all groups combined and according to Hispanic/Latino background. In addition, the distributions of study population characteristics age-standardized to the 2010 US census age distribution ${ }^{45}$ were estimated for periodontitis (CAL $\geq 3 \mathrm{~mm}$ and PD $\geq 4 \mathrm{~mm}$ ) and suspected NAFLD using weighted least squares survey regression. Design-adjusted Wald chi-square tests assessed the associations of categorical variables with periodontitis (CAL $\geq 3 \mathrm{~mm}$ and PD $\geq 4 \mathrm{~mm}$ ) and suspected NAFLD. Potential effect measure modification (EMM) of the association between periodontitis and suspected NAFLD was assessed using design-adjusted Wald chi-square tests comparing models with and without product interaction terms between periodontitis and Hispanic/Latino background, and between periodontitis and gender. Threshold for statistically significant interaction was set at $\mathrm{p}<0.10$. Separate survey logistic regression models for suspected NAFLD based serum transaminases (AST and ALT) thresholds and the FLI $760 \%$ were used to estimate prevalence odds ratios (POR) and 95\% confidence intervals (CI). The first model was stratified by Hispanic/Latino background but did not include other covariates. Subsequent stratified models sequentially adjusted for age and gender and then for NAFLD risk factors (i.e., abdominal obesity, diabetes), smoking, physical activity, educational attainment and acculturation score. Statistical tests were 2 -sided and significance was set at $\mathrm{p}<0.05$. Statistical tests and data analysis were conducted in SAS v. 9.4 (SAS Institute Cary, NC) accounting for the complex sampling design and applying weights to account for the unequal sampling probabilities.

\section{Results}

The overall mean age (SE) was $40.4(0.26)$ years and was greater for Cuban background participants [mean $(\mathrm{SE})=44.9(0.61)$ years] than for any other Hispanic/Latino background groups. The mean acculturation score was highest for Puerto Rican background group (mean=3.15) in comparison to all groups combined (mean=2.07) and about half of the Cuban background group and South-American background group had more than a high school education. A majority of the study participants were never smokers (65.5\%) and a sizable proportion (44.9\%) engaged in some form of moderate physical activity (Table 1).

The overall age standardized percentage of study participants with $<30 \%$ of sites with CAL $\geq 3 \mathrm{~mm}$ or PD $\geq 4 \mathrm{~mm}$ was $53.5 \%$ and $58.6 \%$ respectively while $16 \%$ and $5.72 \%$ respectively had $\geq 30 \%$ of sites with CAL $\geq 3 \mathrm{~mm}$ or PD $\geq 4 \mathrm{~mm}$. The distribution of extent of sites with $\mathrm{CAL} \geq 3 \mathrm{~mm}$ and PD $\geq 4 \mathrm{~mm}$ was similar for each of the covariates explored in this report. For 
example, having $<30 \%$ of sites affected by CAL $\geq 3 \mathrm{~mm}$ and PD $\geq 4 \mathrm{~mm}$ was most common for these covariates (Table 2).

The overall age-standardized prevalence $(95 \% \mathrm{CI})$ of suspected NAFLD was $18.1 \%(17.1 \%-$ $19.0 \%$ ) while $47.1 \%(45.5 \%-48.7 \%)$ met the criteria for FLI $760 \%$. The age-standardized prevalence of suspected NAFLD was greatest among Puerto Ricans and Dominicans background groups at approximately $20 \%$ in each group and, lowest among participants $₫ 65$ years, [10.4\% (95\% CI: 7.48\%, 13.4\%)]. While there was no difference in prevalence of suspected NAFLD (based on serum transaminase levels) according to education and physical activity level, the prevalence of FLI $\geq 60 \%$ differed significantly according to levels of these covariates (Table 3).

There was no significant effect measure modification between periodontitis and gender $(\mathrm{p}=0.2)$ with respect to suspected NAFLD prevalence, hence results were not stratified according to gender. There was no statistically significant difference in the unadjusted and covariate-adjusted associations between periodontitis and suspected NAFLD within each Hispanic/Latino background groups $(\mathrm{p}=0.7$ ). For all groups combined, there was a marginal but statistically non-significant association between the proportion of sites with CAL $23 \mathrm{~mm}$ and suspected NAFLD but this association was attenuated upon adjusting for covariates (Table 4). When periodontitis was defined as the proportion of sites with PD $\geq 4 \mathrm{~mm}$, there was a dose-response association with suspected NAFLD for all groups combined, whereby participants with $230 \%$ of sites affected had an unadjusted POR of 1.39 (95\% CI: 1.02, 1.90 ), while those with $<30 \%$ had an unadjusted POR of 1.19 (95\% CI: 1.03, 1.38).

However, these crude estimates were likewise attenuated towards the null upon covariate adjustment (Table 5). Similar patterns in unadjusted and covariate-adjusted estimates for CAL $23 \mathrm{~mm}$ were observed overall among all study participants, when the FLI was used as the outcome (see supplementary Table 1 in online Journal of Periodontology). For PD $\geq 4 \mathrm{~mm}$, the observed positive unadjusted dose-response relationship persisted upon covariate adjustment but was statistically non-significant in the fully adjusted model (see supplementary Table 2 in online Journal of Periodontology).

\section{Discussion}

This cross-sectional study of Hispanic/Latino adults found no meaningful association between extent of periodontitis and indicators of NAFLD. These findings are inconsistent with previous studies in animal models ${ }^{31,32}$ and human studies ${ }^{31,33,46}$ that have reported positive associations. While the unadjusted estimates of this association were greater for the fatty liver index than for serum transaminases, these estimates were attenuated upon covariate adjustment. The FLI was developed and tested for accuracy in a population of Europeans ${ }^{40}$ and may not be optimal for a population of Hispanics/Latinos. Furthermore, the algorithm for the FLI includes waist circumference, which was adjusted for as a NAFLD risk factor and thus adjusted estimates for the FLI may represent a form of over-adjustment bias. A sensitivity analysis removing waist circumference as a covariate from the fullyadjusted model for the FLI resulted in POR within the entire study population of 1.13 (95\% CI: $1.01,1.27)$ and $1.31(95 \%$ CI: $1.03,1.68)$ for PD $<30 \%$ and $330 \%$ respectively and POR of 0.98 (95\% CI: $0.84,1.14)$ and 0.98 (95\% CI: $0.79,1.21)$ for CAL $<30 \%$ and $330 \%$ 
respectively. Given that the prevalence of suspected NALFD of $18.1 \%$ based on transaminases differs significantly from the $47.1 \%$ for FLI $\nsucceq 60 \%$ (Table 3), a threshold of FLI $\checkmark 60 \%$ may not be optimal for this population.

NAFLD is a heterogeneous condition and while its risk factors appear universal, the contribution of these risk factors to the burden of NAFLD might differ among population groups. Specifically, while the prevalence of NAFLD is reported to be greater among Hispanic/Latino populations compared to other racial/ethnic groups,${ }^{47}$ factors such as periodontitis investigated in this study represents an upstream (i.e. distal) risk factor whose effects were likely rendered non-significant by more proximal risk factors, hence the appearance of a null association in this study.

Other likely reasons for the discrepancies between current and prior findings include the method for characterizing the outcome. While liver ultrasound is the preferred method for characterizing NAFLD in epidemiologic settings, this investigation unlike prior studies, relied primarily on serum transaminases (AST, ALT) and the FLI for characterizing NAFLD. Serum transaminases are non-specific markers on hepatocellular injury and are not always elevated when NAFLD is present. Therefore, potential for misclassification of true NAFLD status is possible in this study. If this misclassification was non-differential according to periodontitis status, the reported estimates were likely biased towards the null, however, if misclassification was differential, then the direction of bias may be hard to predict. Furthermore, the FLI was developed for predicting the likelihood of having NAFLD and may be unsuitable for epidemiologic studies.

While we attempted to minimize misclassification of NAFLD cases by excluding individuals who reported excessive alcohol consumption, due of the non-specific nature of serum transaminases, it is also possible that other hepatic conditions might be responsible for elevated serum transaminase levels. Given the self-reported nature of alcohol use, potential for misreporting and misclassification of alcohol use status is also likely and could have biased these findings.

Lastly, possible racial/ethnic differences may exist with regards to the relationship between periodontitis and NAFLD, whereby the profile of NAFLD risk factors, such as lifestyle and diet, genetics or the intestinal microbiome may differ in their associations with periodontitis among groups who self-identified by race or ethnicity. Within the constraints of our characterization of NAFLD, it is thus important to report on the lack of an association between periodontitis and NAFLD among individuals of Hispanic/Latino background.

Study limitations include the cross-sectional design that is subject to incidence-prevalence bias. Serum transaminases are not always elevated when NAFLD is present and so outcome definition may have been underestimated and/or misclassified. The FLI does not accurately capture NAFLD because it only states the likelihood of having the condition. In spite of these limitations, study strengths include the large study sample and the first report to investigate this question of concerning public health in a diverse group of Hispanics/Latinos. Furthermore, the population of Hispanics/Latinos studied here also sets this study apart from 
previous epidemiologic studies that have investigated periodontitis and NAFLD in Japanese $^{31}$ and German ${ }^{33}$ populations.

\section{Conclusions}

Previously reported associations between periodontitis and NAFLD were not replicated in this study of a diverse group of Hispanic/Latino men and women. One reason for this discrepancy might be the use of transaminases to characterize NAFLD in this study compared to liver ultrasounds and biopsies used in prior studies. ${ }^{31}$

\section{Supplementary Material}

Refer to Web version on PubMed Central for supplementary material.

\section{Acknowledgments}

Grant support: Support for this work was provided by the National Institutes of Health/National Institute of Dental and Craniofacial Research (grant R03DE025652-01A1).

The authors thank the staff and participants of HCHS/SOL for their important contributions. The Hispanic Community Health Study/Study of Latinos was carried out as a collaborative study supported by contracts from the National Heart, Lung, and Blood Institute (NHLBI) to the University of North Carolina (N01-HC65233), University of Miami (N01-HC65234), Albert Einstein College of Medicine (N01-HC65235), Northwestern University (N01HC65236), and San Diego State University (N01-HC65237). The following Institutes/Centers/Offices contribute to the HCHS/SOL through a transfer of funds to the NHLBI: National Institute on Minority Health and Health Disparities, National Institute on Deafness and Other Communication Disorders, National Institute of Dental and Craniofacial Research, National Institute of Diabetes and Digestive and Kidney Diseases, National Institute of Neurological Disorders and Stroke, NIH Institution-Office of Dietary Supplements.

\section{References Cited}

1. Angulo P. Nonalcoholic fatty liver disease. N Engl J Med. 2002; 346:1221-1231. [PubMed: 11961152]

2. Clark JM, Brancati FL, Diehl AM. Nonalcoholic fatty liver disease. Gastroenterology. 2002; 122:1649-1657. [PubMed: 12016429]

3. Erbey JR, Silberman C, Lydick E. Prevalence of abnormal serum alanine aminotransferase levels in obese patients and patients with type 2 diabetes. Am J Med. 2000; 109:588-590. [PubMed: 11063962]

4. Ruhl CE, Everhart JE. Determinants of the association of overweight with elevated serum alanine aminotransferase activity in the United States. Gastroenterology. 2003; 124:71-79. [PubMed: 12512031]

5. Musso G, Gambino R, Cassader M, Pagano G. Meta-analysis: natural history of non-alcoholic fatty liver disease (NAFLD) and diagnostic accuracy of non-invasive tests for liver disease severity. Ann Med. 2011; 43:617-649. [PubMed: 21039302]

6. Baumeister SE, Volzke H, Marschall P, et al. Impact of fatty liver disease on health care utilization and costs in a general population: a 5-year observation. Gastroenterology. 2008; 134:85-94. [PubMed: 18005961]

7. Clark JM. The epidemiology of nonalcoholic fatty liver disease in adults. J Clin Gastroenterol. 2006; 40:S5-10. [PubMed: 16540768]

8. Marchesini G, Brizi M, Morselli-Labate AM, et al. Association of nonalcoholic fatty liver disease with insulin resistance. Am J Med. 1999; 107:450-455. [PubMed: 10569299]

9. Grundy SM, Brewer HB Jr, Cleeman JI, Smith SC Jr, Lenfant C. Definition of metabolic syndrome: Report of the National Heart, Lung, and Blood Institute/American Heart Association conference on scientific issues related to definition. Circulation. 2004; 109:433-438. [PubMed: 14744958] 
10. Kugelmas M, Hill DB, Vivian B, Marsano L, McClain CJ. Cytokines and NASH: a pilot study of the effects of lifestyle modification and vitamin E. Hepatology (Baltimore, Md). 2003; 38:413419.

11. Hui JM, Hodge A, Farrell GC, Kench JG, Kriketos A, George J. Beyond insulin resistance in NASH: TNF-alpha or adiponectin? Hepatology (Baltimore, Md). 2004; 40:46-54.

12. Saab S, Manne V, Nieto J, Schwimmer JB, Chalasani NP. Nonalcoholic Fatty Liver Disease in Latinos. Clin Gastroenterol Hepatol. 2016; 14:5-12. [PubMed: 25976180]

13. Pan JJ, Fallon MB. Gender and racial differences in nonalcoholic fatty liver disease. World $\mathrm{J}$ Hepatol. 2014; 6:274-283. [PubMed: 24868321]

14. Eklund SA, Burt BA. Risk factors for total tooth loss in the United States; longitudinal analysis of national data. J Public Health Dent. 1994; 54:5-14. [PubMed: 8164192]

15. Burt BA, Eklund SA. Dentistry, Dental Practice and the Community. Elsevier Inc; 2005.

16. Eke PI, Dye BA, Wei L, Thornton-Evans GO, Genco RJ. Prevalence of periodontitis in adults in the United States: 2009 and 2010. J Dent Res. 2012; 91:914-920. [PubMed: 22935673]

17. Papapanou PN. The prevalence of periodontitis in the US: forget what you were told. J Dent Res. 2012; 91:907-908. [PubMed: 22935674]

18. Su Y, Wang D, Xuan D, et al. Periodontitis as a novel contributor of adipose tissue inflammation promotes insulin resistance in a rat model. J Periodontol. 2013; 84:1617-1626. [PubMed: 23289867]

19. Watanabe K, Petro BJ, Shlimon AE, Unterman TG. Effect of periodontitis on insulin resistance and the onset of type 2 diabetes mellitus in Zucker diabetic fatty rats. J Periodontol. 2008; 79:12081216. [PubMed: 18597603]

20. Gurav AN. Periodontitis and insulin resistance: casual or causal relationship? Diabetes Metab J. 2012; 36:404-411. [PubMed: 23275933]

21. Genco RJ, Grossi SG, Ho A, Nishimura F, Murayama Y. A proposed model linking inflammation to obesity, diabetes, and periodontal infections. J Periodontol. 2005; 76:2075-2084.

22. Benguigui C, Bongard V, Ruidavets JB, et al. Metabolic syndrome, insulin resistance, and periodontitis: a cross-sectional study in a middle-aged French population. J Clin Periodontol. 2010; 37:601-608. [PubMed: 20492076]

23. Timonen P, Suominen-Taipale L, Jula A, Niskanen M, Knuuttila M, Ylostalo P. Insulin sensitivity and periodontal infection in a non-diabetic, non-smoking adult population. J Clin Periodontol. 2011; 38:17-24.

24. Allen EM, Matthews JB, DJOH, Griffiths HR, Chapple IL. Oxidative and inflammatory status in Type 2 diabetes patients with periodontitis. J Clin Periodontol. 2011; 38:894-901. [PubMed: 21883360]

25. Ebersole JL, Cappelli D, Mathys EC, et al. Periodontitis in humans and non-human primates: oralsystemic linkage inducing acute phase proteins. Ann Periodontol. 2002; 7:102-111. [PubMed: 16013223]

26. Beck JD, Offenbacher S. The association between periodontal diseases and cardiovascular diseases: a state-of-the-science review. Ann Periodontol. 2001; 6:9-15. [PubMed: 11887476]

27. Slade GD, Offenbacher S, Beck JD, Heiss G, Pankow JS. Acute-phase inflammatory response to periodontal disease in the US population. J Dent Res. 2000; 79:49-57. [PubMed: 10690660]

28. Wu T, Trevisan M, Genco RJ, Falkner KL, Dorn JP, Sempos CT. Examination of the relation between periodontal health status and cardiovascular risk factors: serum total and high density lipoprotein cholesterol, C-reactive protein, and plasma fibrinogen. Am J Epidemiol. 2000; 151:273-282. [PubMed: 10670552]

29. Ebersole JL, Machen RL, Steffen MJ, Willmann DE. Systemic acute-phase reactants, C-reactive protein and haptoglobin, in adult periodontitis. Clin Exp Immunol. 1997; 107:347-352. [PubMed: 9030874]

30. Slade GD, Ghezzi EM, Heiss G, Beck JD, Riche E, Offenbacher S. Relationship between periodontal disease and C-reactive protein among adults in the Atherosclerosis Risk in Communities study. Arch Intern Med. 2003; 163:1172-1179. [PubMed: 12767953] 
31. Yoneda M, Naka S, Nakano K, et al. Involvement of a periodontal pathogen, Porphyromonas gingivalis on the pathogenesis of non-alcoholic fatty liver disease. BMC Gastroenterol. 2012; 12:16. [PubMed: 22340817]

32. Dos Santos Carvalho J, Cardoso Guimarães Vasconcelos AC, Herlany Pereira Alves E, et al. Steatosis caused by experimental periodontitis is reversible after removal of ligature in rats. J Perio Res. 2017; 52:883-892.

33. Akinkugbe AA, Slade GD, Sidney Barritt A, et al. Periodontitis and Non-alcoholic Fatty Liver Disease, a population based cohort investigation in the Study of Health in Pomerania. J Clin Periodontol. 2017; 44:1077-1087. [PubMed: 28804947]

34. Sorlie PD, Aviles-Santa LM, Wassertheil-Smoller S, et al. Design and implementation of the Hispanic Community Health Study/Study of Latinos. Ann Epidemiol. 2010; 20:629-641. [PubMed: 20609343]

35. Lavange LM, Kalsbeek WD, Sorlie PD, et al. Sample design and cohort selection in the Hispanic Community Health Study/Study of Latinos. Ann Epidemiol. 2010; 20:642-649. [PubMed: 20609344]

36. Daviglus ML, Talavera GA, Aviles-Santa ML, et al. Prevalence of major cardiovascular risk factors and cardiovascular diseases among Hispanic/Latino individuals of diverse backgrounds in the United States. JAMA. 2012; 308:1775-1784. [PubMed: 23117778]

37. Sanders AE, Campbell SM, Mauriello SM, et al. Heterogeneity in periodontitis prevalence in the Hispanic Community Health Study/Study of Latinos. Ann Epidemiol. 2014; 24:455-462. [PubMed: 24731697]

38. Carlos JP, Wolfe MD, Kingman A. The extent and severity index: a simple method for use in epidemiologic studies of periodontal disease. J Clin Periodontol. 1986; 13:500-505. [PubMed: 3459740]

39. Clark JM, Brancati FL, Diehl AM. The prevalence and etiology of elevated aminotransferase levels in the United States. Am J Gastroenterol. 2003; 98:960-967. [PubMed: 12809815]

40. Bedogni G, Bellentani S, Miglioli L, et al. The Fatty Liver Index: a simple and accurate predictor of hepatic steatosis in the general population. BMC Gastroenterol. 2006; 6:33. [PubMed: 17081293]

41. American Diabetes Association. Diagnosis and classification of diabetes mellitus. Diabetes care. 2012; 35(Suppl 1):S64-71. [PubMed: 22187472]

42. Marin G, Sabogal F, Marin BV, Otero-Sabogal R, Perez-Stable EJ. Development of a Short Acculturation Scale for Hispanics. Hisp J Behav Sci. 1987; 9:183-205.

43. Kallwitz ER, Daviglus ML, Allison MA, et al. Prevalence of suspected nonalcoholic fatty liver disease in Hispanic/Latino individuals differs by heritage. Clin Gastroenterol Hepatol. 2015; 13:569-576. [PubMed: 25218670]

44. Bull FC, Maslin TS, Armstrong T. Global physical activity questionnaire (GPAQ): nine country reliability and validity study. J Phys Act Health. 2009; 6:790-804. [PubMed: 20101923]

45. Ennis SR, Ríos-Vargas M, Albert NG. Census Briefs. Washington: U.S. Census Bureau; 2011. The Hispanic Population: 2010.

46. Alazawi W, Bernabe E, Tai D, et al. Periodontitis is associated with significant hepatic fibrosis in patients with non-alcoholic fatty liver disease. PLoS One. 2017; 12:e0185902. [PubMed: 29220367]

47. Browning JD, Szczepaniak LS, Dobbins R, et al. Prevalence of hepatic steatosis in an urban population in the United States: impact of ethnicity. Hepatology. 2004; 40:1387-1395. [PubMed: 15565570] 


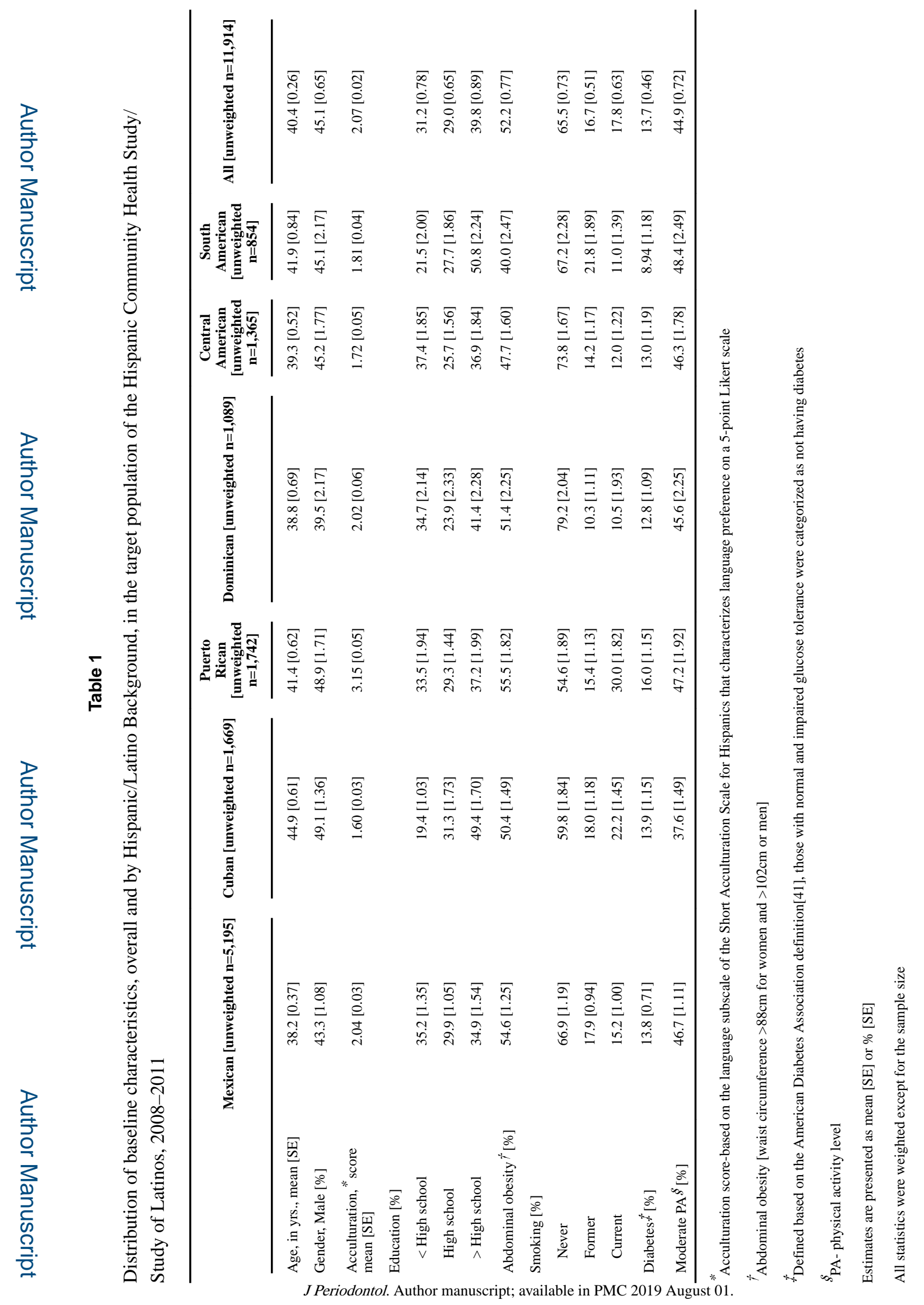




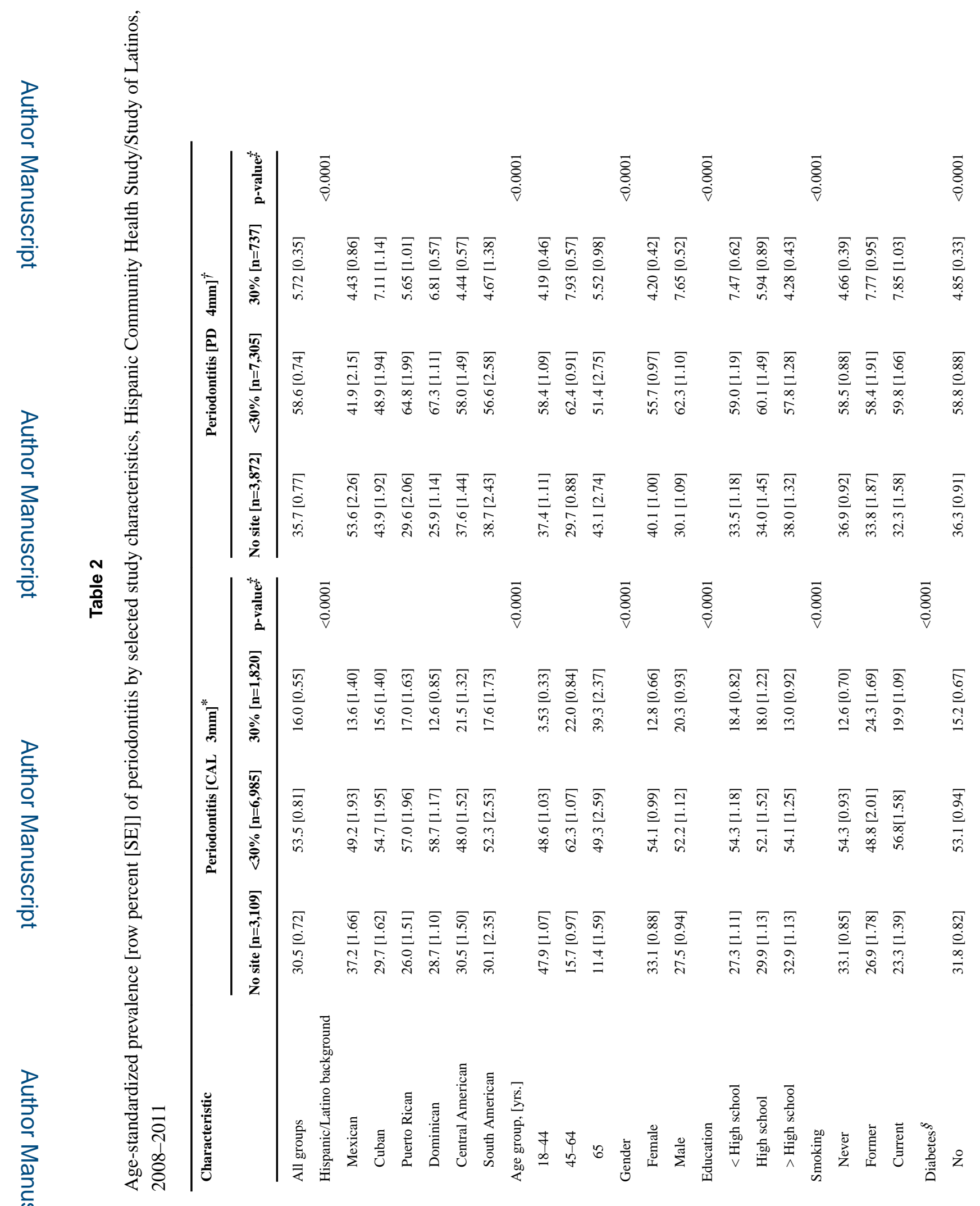

J Periodontol. Author manuscript; available in PMC 2019 August 01. 


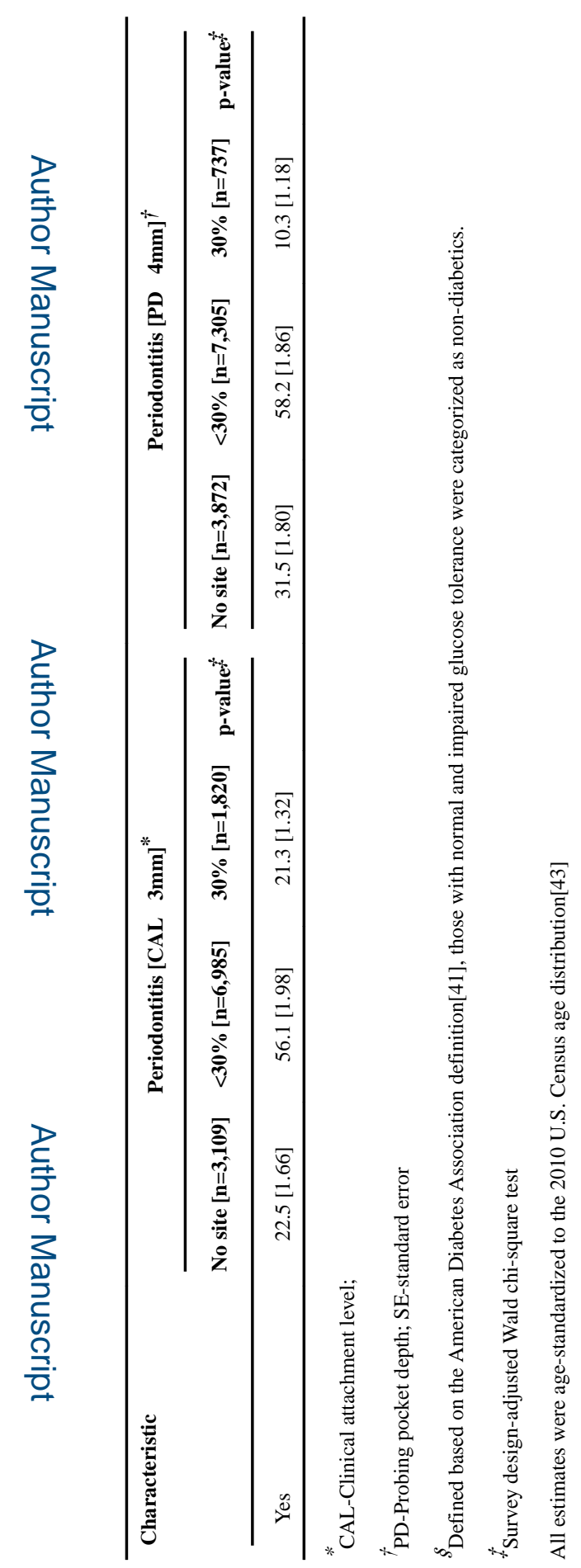

로을

J Periodontol. Author manuscript; available in PMC 2019 August 01. 


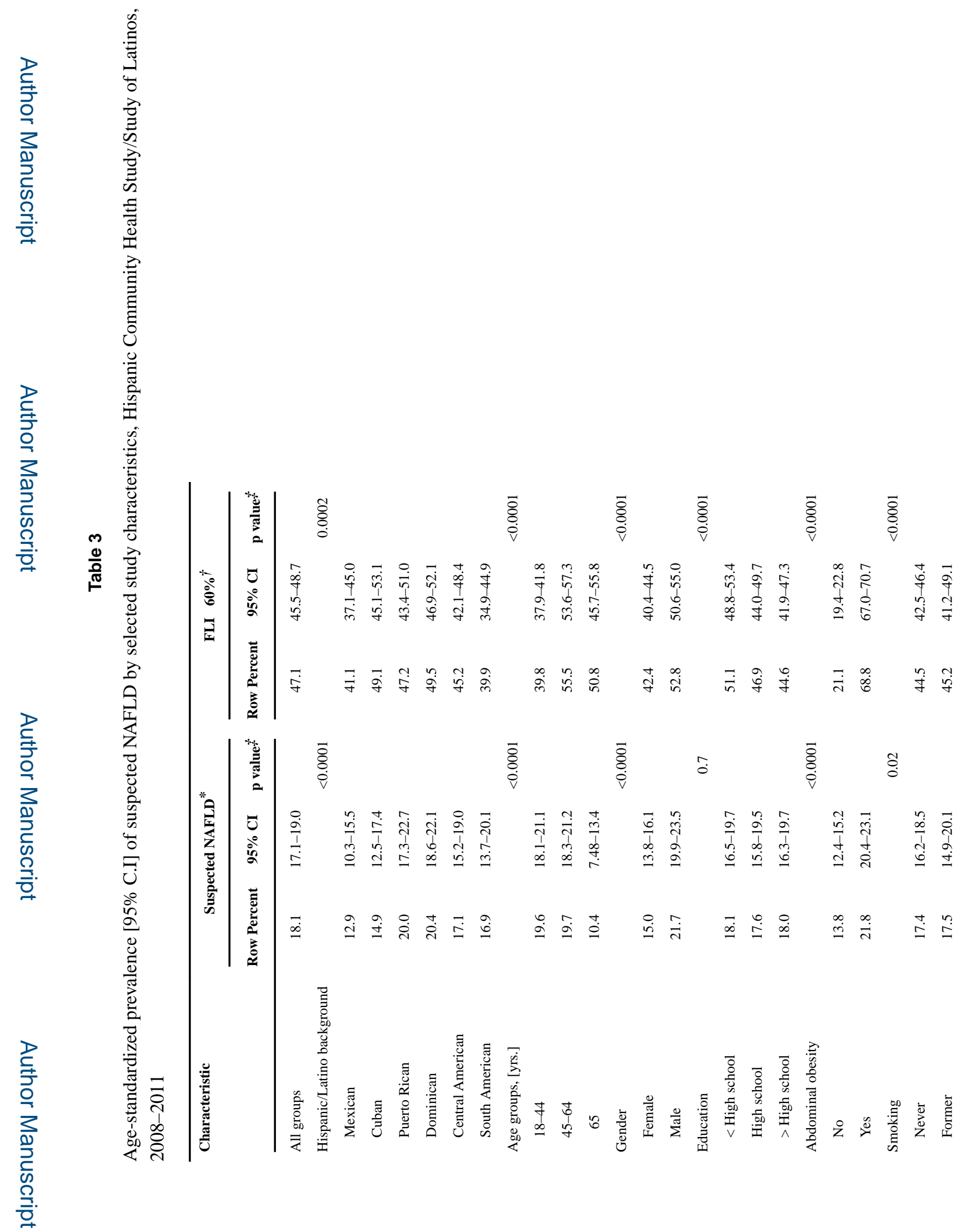

J Periodontol. Author manuscript; available in PMC 2019 August 01. 


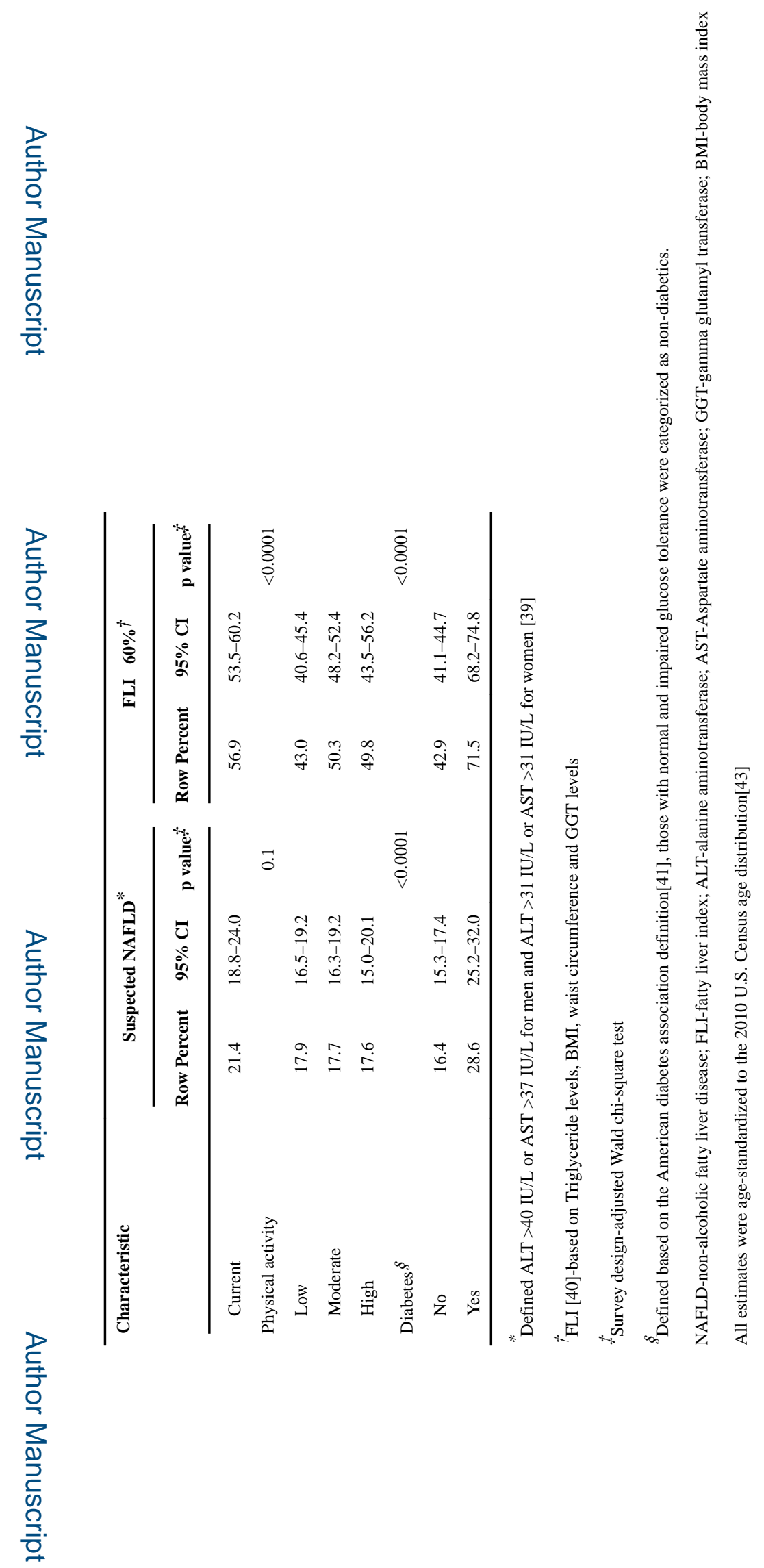

J Periodontol. Author manuscript; available in PMC 2019 August 01. 


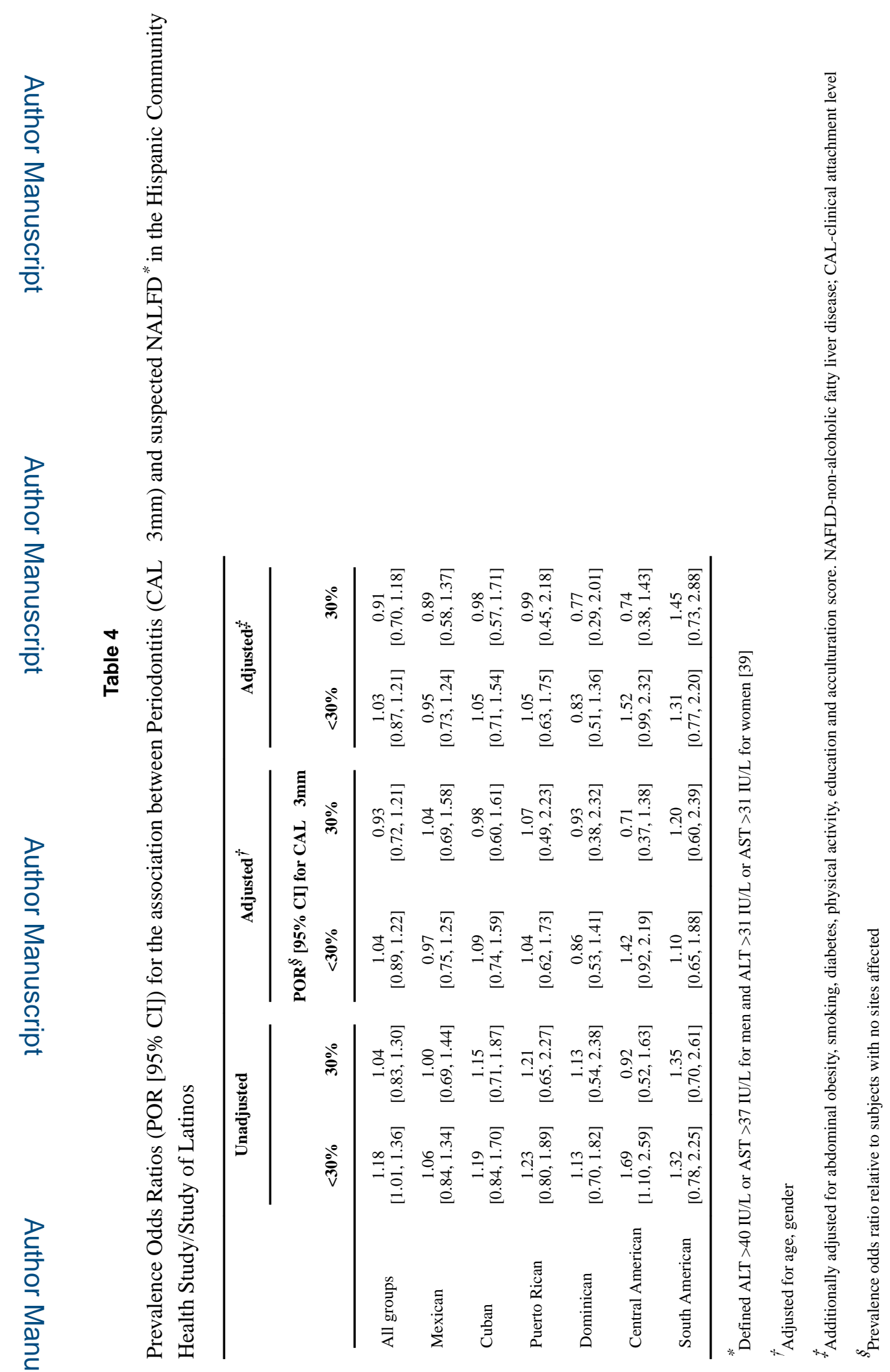

J Periodontol. Author manuscript; available in PMC 2019 August 01. 


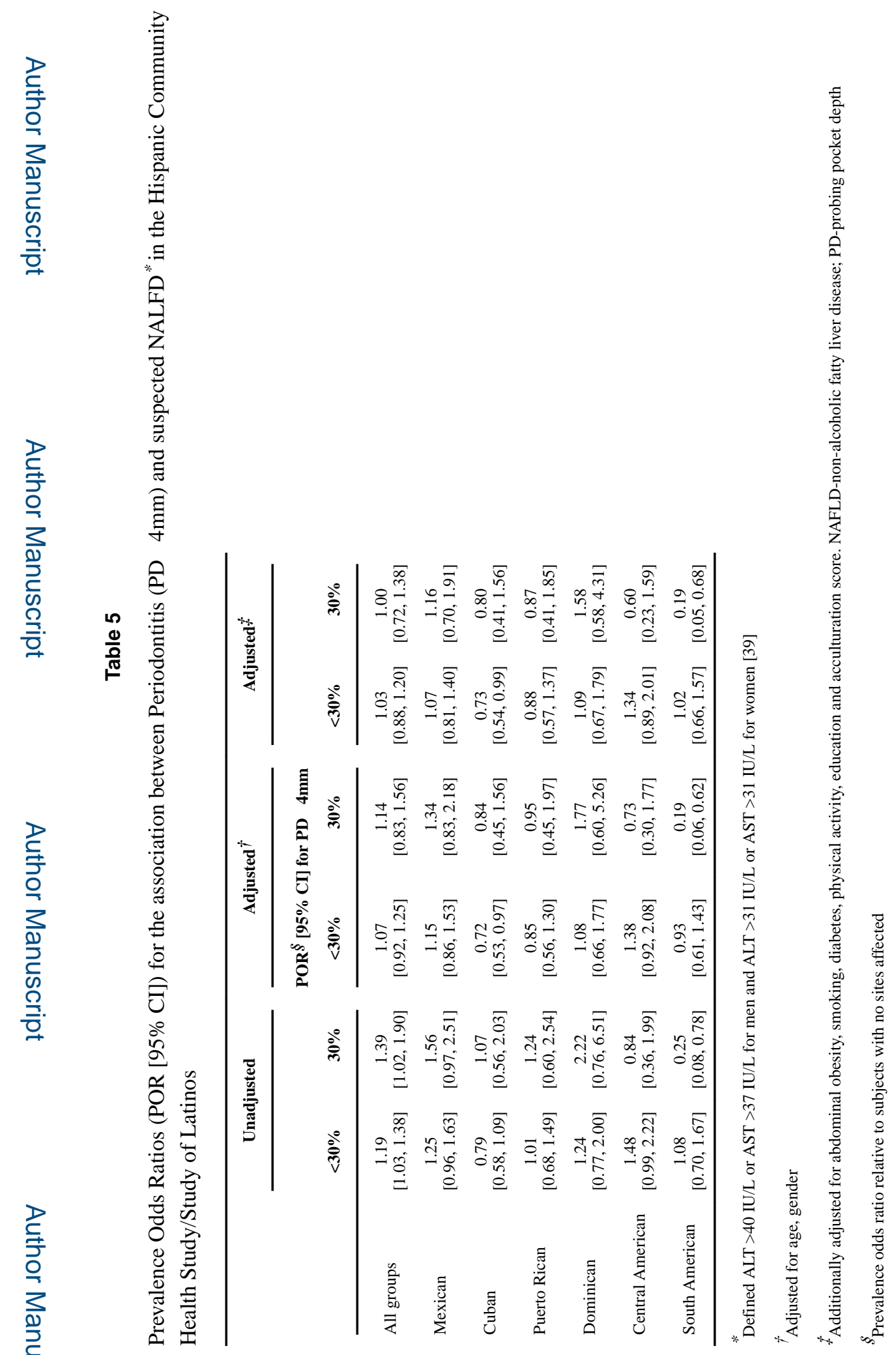

